\title{
ESTIMATION OF EQUIVALENCY UNITS FOR VEHICLE TYPES UNDER MIXED TRAFFIC CONDITIONS: MULTIPLE NON-LINEAR REGRESSION APPROACH
}

\author{
Seelam Srikanth ${ }^{1 *}$, Arpan Mehar ${ }^{1}$ \\ ${ }^{1}$ Department of Civil engineering, National Institute of Technology, Warangal, Telangana, 506004, \\ India
}

(Received: November 2016 / Revised: May 2017 / Accepted: October 2017)

\begin{abstract}
The accuracy of measured traffic flow on a roadway largely depends on the correctness of the PCU factors used for converting traffic counts. PCU is the number of passenger cars that are displaced by a single heavy vehicle of a particular type under prevailing roadway, traffic and control conditions. The aim of the present study is to develop more appropriate models for estimating the equivalency units of different vehicle types on multilane highways, considering the limitations of available methods. Estimation of equivalency units for vehicle types is described by developing speed models based on multiple non-linear regression approaches. The equivalency units estimated by using models are found to be realistic and logical under heterogeneous traffic flow conditions. The PCU values estimated by the multiple non-linear regression method are compared with and found to be relatively higher values than the values obtained by the dynamic PCU. The accuracy of the models is checked by comparing the observed values of speed with estimated speeds. The multiple non-linear regression approach is also used for estimating the equivalency units on six-lane divided highways. Results indicate that the proposed methodology can be used for estimation of equivalency units for vehicle types under mixed traffic conditions.
\end{abstract}

Keywords: Equivalency units; Multiple non-linear regression; PCU; Speed

\section{INTRODUCTION}

Classical macroscopic models, which were developed for the modelling of traffic flow, considered traffic to be homogeneous and lane-based. In a homogeneous and lane-based traffic system, all vehicles are of the same type, moving strictly in lanes and at nearly the same speed, a pattern which is more prevalent in developed countries. However, in developing countries like India, the traffic conditions are heterogeneous and non-lane-based in nature. In heterogeneous and non-lane-based traffic conditions, multiple types of vehicles with different static and dynamic characteristics share the same carriageway without any physical separation between them. Because of these complexities, the variations in traffic stream behavior are very high. In order to examine the heterogeneous traffic flow, researchers proposed different techniques and models. One of the most widely used techniques at the macroscopic level is to homogenize the traffic before modelling the traffic flow. Passenger Car Unit (PCU) has been proposed by various researchers for different classes of vehicles to homogenize the heterogeneous traffic.

The idea of PCU was first introduced in the Highway Capacity Manual (HCM) in 1965 to

\footnotetext{
*Corresponding author's email: ssseelamsrikanth@gmail.com, Tel: +91-94-9130-3992, Fax: +91-870-2459547 Permalink/DOI: https://doi.org/10.14716/ijtech.v8i5.864
} 
account for the effect of trucks and buses in the traffic stream. The HCM is a publication of the Transportation Research Board of the National Academies of Science in the United States. It contains concepts, guidelines, and computational procedures for computing the capacity and quality of service of various highway facilities including freeways, highways, arterial roads, roundabouts, signalized intersections and un-signalized intersections. Subsequently, PCU values of vehicle type and estimation have been a subject of interest all over the world. The accuracy of measured traffic flow on a roadway largely depends on the correctness of PCU factors used for converting traffic counts. The PCU of a vehicle type depends on vehicular characteristics, stream characteristics, roadway characteristics, environmental factors, climate conditions, and control conditions (Chandra et al., 1995; Karim et al., 1999).

Several methods were developed for determining PCU values such as modified density method, speed, and area ratio method, the method based on relative delay, headway method, multiple linear regression method, and simulation method. However, most of the methods are unsuitable under highly heterogeneous traffic conditions. As per the literature review (Chandra et al., 1995), the dynamic PCU method and homogenization coefficient method are commonly used for estimating PCU values of various vehicle types under mixed traffic conditions. In the homogenization coefficient method, PCU values are estimated by the ratio of the average speed and length of vehicle type. However, Indian traffic follows a disordered lane system, so using the speed of the vehicle type with length alone may not be appropriate, as the width of vehicle types also varies. In the dynamic PCU method, a rectangular projected area is used instead of using the length of vehicle types. The method provides excellent results under all kinds of roadway and traffic conditions. Hence, the dynamic method of estimating PCUs is considered useful for all types of vehicles under various traffic conditions. The dynamic method considers the speed ratio and an area ratio of vehicle types but ignores the effect of headways maintained by vehicles. Traffic composition is another variable which influences the PCU values under heterogeneous traffic conditions. Therefore, it is necessary to develop a more appropriate model for estimating PCU values of different types of vehicles on multilane highways, such as those prevailing in India.

Following the introduction of the term PCU in the HCM, considerable research effort has been made toward the estimation of PCU values under various roadway conditions. For example, John and Kobett (1978) developed a non-linear relationship for deriving PCU values by using the mean speed as the measure of equivalence, and concluded that PCU values vary not only with different roadways conditions but also with different traffic control conditions. Huber (1982) introduced the concept of equal density to relate mixed traffic flow rate and base flow rate in a calculation of PCU. The observations were made with two different traffic streams, one that had trucks mixed with passenger cars and the other that had passenger cars only, and the impedance was measured as a function of traffic flow. Three measures of impedance were considered, each of which generate a separate PCU value for a truck of given physical and operational characteristics. The PCU values are related to the ratio between the volumes of the two streams at common levels of impedance. Cunagin and Messer (1982) developed an analytical method to estimate PCU values for 14 different vehicle types under different traffic conditions on both two-lane and rural four-lane highways in several states. In this study, relative delays were used to estimate the PCUs.

Chandra et al. (1995) proposed a method for estimating PCU values of different vehicle types under mixed traffic conditions. The basic concept used in this method is that the PCU value of a subject vehicle is directly proportional to the speed ratio and inversely proportional to the projected area ratio of the standard car to the subject vehicle type. Chandra et al. (1995) also developed speed models for vehicle types under heterogeneous traffic conditions by considering the average speed of vehicles and the traffic volume as independent variables. Chandra and 
Kumar (2003) further studied the effect of road width on PCU values estimated on two-lane highways and observed that the PCU of a vehicle type increases with the increase in carriageway width as they get more freedom on the wider roads.

Cao and Sano (2012) investigated an accurate methodology for estimating motorcycle equivalency units (MEUs) under mixed traffic flow conditions by considering speed, the physical size of the subject vehicle, and the surrounding motorcycles. Field data was collected in Hanoi, capital of Vietnam and Equation 1 was proposed. The results indicate that the MEU values of vehicle types car, bus, minibus, and bicycle are estimated as 3.4, 10.5, 8.3, and 1.4, respectively.

$$
\mathrm{MEU}_{\mathrm{k}}=\left(\mathrm{V}_{\mathrm{mc}} / \mathrm{V}_{\mathrm{k}}\right) *\left(\mathrm{~S}_{\mathrm{k}} / \mathrm{S}_{\mathrm{mc}}\right)
$$

where, MEU $\mathrm{k}_{\mathrm{k}}$ is the MEU of vehicle type $\mathrm{k}, \mathrm{V}_{\mathrm{mc}}$ and $\mathrm{V}_{\mathrm{k}}$ are the mean speed of the motorcycles and vehicle type $k$, respectively $(\mathrm{m} / \mathrm{s})$, and $S_{\mathrm{mc}}$ and $S_{\mathrm{k}}$ are the mean effective spacing $\left(\mathrm{m}^{2}\right)$ of motorcycles and vehicle type $\mathrm{k}$, respectively.

Webster and Elefteriadou (1999) conducted a simulation study to develop a method for estimating truck-passenger car equivalents on freeways. This research developed truckpassenger car equivalents using traffic flow simulation based on traffic density. Arasan and Arkatkar (2010) also analyzed the effects of traffic volume and road width on the PCU of vehicles under heterogeneous conditions using the microscopic simulation technique. Simulation model HETEROSIM was used to study the PCU over a wide range of traffic volumes.

The objective of the present study is to develop more appropriate models for estimating equivalency units of different vehicle types on multilane highways under heterogeneous traffic conditions by moving beyond the limitations of available methods.

\section{METHODOLOGY}

The methodology for estimation of PCU is to develop a speed model consisting of multiple independent variables based on the non-linear regression method. The equation consists of variables like the proportion of all vehicle types, an average speed of vehicle types other than small car (CS), where CS is consider as standard vehicle, and area ratios of CS to all other vehicle types. These are considered independent variables that influence the average speed of the CS. The product of the area ratio of CS to subject vehicle type, proportion share of subject vehicle type, and average speed of subject vehicle type are used as a multiplicative components, whereas a proportional share of CS is used as an additive component in the proposed equation. The proposed regression model was developed to predict the speed of standard vehicle types, whose coefficients are estimated as equivalency units of all subject vehicle types. Equation 2 is provided by the basic equation for predicting the average speed of standard car.

$$
V_{C S}=\sum_{j=1}^{k} a_{j}\left(\frac{A_{C S}}{A_{j}} \times n_{j} \times V_{j}\right)+a_{i} \times n_{C S}
$$

where $V_{C S}$ is the average speed of small car $(\mathrm{km} / \mathrm{h}), a_{j}$ and $a_{i}$ are the regression coefficients, $k$ is total number of vehicle types in the traffic stream, $V_{j}$ is average speed of vehicle type $\mathrm{j}(\mathrm{km} / \mathrm{h})$, $n_{j}$ is the proportion of vehicle type $\mathrm{j}, n_{c s}$ is proportion of small cars, $A_{j}$ is the rectangular projected area of subject vehicle type $\mathrm{j}$, and $A_{c s}$ is the rectangular projected area of a small car $\left(\mathrm{m}^{2}\right)$. 
The intercept term in the equation was not kept because the speed of the small car type must be fully explained by the chosen variables. The PCU value of vehicle type $j$ is the regression coefficient of corresponding vehicle type $\left(\mathrm{a}_{\mathrm{j}}\right)$. Similarly, the Equations 3 and 4 are also proposed in case of two-wheeler (TW) and heavy commercial (HCV) vehicle types, in order to obtain their average speeds and to estimate equivalency units in their respective term.

$$
V_{T W}=\sum_{j=1}^{k} b_{j}\left(\frac{A_{T W}}{A_{j}} \times n_{j} \times V_{j}\right)+b_{i} \times n_{T W}
$$

where $V_{T W}$ is the average speed of two-wheelers $(\mathrm{km} / \mathrm{h}), b_{j}$ and $b_{i}$ are the regression coefficients, $k$ is total number of vehicle types in the traffic stream, $V_{j}$ is average speed of vehicle type $\mathrm{j}$ in $\mathrm{km} / \mathrm{h}, n_{j}$ is the proportion of vehicle type $\mathrm{j}, n_{T W}$ is the proportion of two-wheelers, $A_{j}$ is the rectangular projected area of subject vehicle type $\mathrm{j}$, and $A_{T W}$ is the rectangular projected area of the two-wheelers.

$$
V_{H C V}=\sum_{j=1}^{k} c_{j}\left(\frac{A_{H C V}}{A_{j}} \times n_{j} \times V_{j}\right)+c_{i} \times n_{H C V}
$$

where $V_{H C V}$ is the average speed of $\mathrm{HCV}(\mathrm{km} / \mathrm{h}), c_{j}$ and $c_{i}$ are the regression coefficients, $k$ is the total number of vehicle types in the traffic stream, $V_{j}$ is the average speed of vehicle type $\mathrm{j}$ $(\mathrm{Km} / \mathrm{h}), n_{j}$ is the proportion of vehicle category $\mathrm{j}, n_{H C V}$ is proportion of $\mathrm{HCV}, A_{j}$ is the rectangular projected area of subject vehicle type $\mathrm{j}$, and $A_{H C V}$ is the rectangular projected area of $\mathrm{HCV}$.

Field data was collected at different mid-block sections of multilane divided intercity highways with plain terrains and straight alignments. Different sections of divided highway were identified and field investigations were performed. Section-I and Section-II were selected on National Highway $(\mathrm{NH}) 163$, and both have $1.5 \mathrm{~m}$ shoulders in each direction of travel. The Section-III was selected from NH 16, a six-lane divided intercity highway that has $1.8 \mathrm{~m}$ paved shoulders. The video graphic method was used for collecting speed and volume data. A trap length of $50 \mathrm{~m}$ was marked on highway sections to estimate the speed of vehicles by noting the travel time. The duration of data collection, traffic volume, and posted speed limits on different highway sections are given in Table 1. Vehicle type surveys were also carried out to obtain the clear dimensions of different vehicle types and are given in Table 2. Traffic volume and speed data were extracted manually from the video recordings playing on a big screen monitor in the traffic engineering laboratory.

Table 1 Duration, traffic volume, and free speed of different sections

\begin{tabular}{ccccc}
\hline \multirow{2}{*}{ Section } & Duration & \multicolumn{2}{c}{ Traffic Volume (Veh/hr) } & $\begin{array}{c}\text { Posted Speed Limit } \\
\text { (km/h) }\end{array}$ \\
\cline { 2 - 3 } Section-I & $\begin{array}{c}\text { 9:00 AM to 12:00 PM } \\
\text { and }\end{array}$ & 1512 & 576 & 80 \\
& $\begin{array}{c}\text { 3:00 PM to 6:00 PM } \\
\text { 9:00 AM to 12:00 PM } \\
\text { and }\end{array}$ & 1400 & 600 & 80 \\
Section-II & $\begin{array}{c}\text { Minimum } \\
\text { 3:00 PM to 6:00 PM }\end{array}$ & & \\
Section-III & $\begin{array}{c}\text { 9:00 AM to 12:00 PM } \\
\text { and }\end{array}$ & 1776 & 900 & 90 \\
\hline
\end{tabular}


Table 2 Clear dimensions of vehicle types and projected area

\begin{tabular}{lccc}
\hline \multicolumn{1}{c}{ Vehicle Type } & Length $(\mathrm{m})$ & Width $(\mathrm{m})$ & Area $\left(\mathrm{m}^{2}\right)$ \\
\hline Standard Car (CS) & 3.72 & 1.44 & 5.36 \\
Big Car (CB) & 4.58 & 1.77 & 8.11 \\
Light Commercial Vehicles (LCV) & 4.30 & 1.56 & 6.71 \\
High Commercial Vehicles (HCV) & 6.70 & 2.30 & 15.41 \\
Multi Axle Vehicles (MAV) & 11.50 & 2.42 & 27.83 \\
Two-Wheeler (TW) & 1.97 & 0.74 & 1.46 \\
Auto (3W) & 3.20 & 1.30 & 4.16 \\
Bus (B) & 10.60 & 2.40 & 25.44 \\
\hline
\end{tabular}

\section{RESULTS AND DISCUSSION}

\subsection{Field Data Analysis}

The field data collected at different highway sections was analyzed by measuring classified volume count and speed at each 5 minute interval. The traffic composition and average speed of all vehicle types on all sections are given in Table 3. Field data collected at Section-I was used for the development of multiple non-linear regression (MNLR) speed models and Section-II and Section-III data was used for the validation of the developed model.

Table 3 Average speed and percentage share of vehicles at study sections

\begin{tabular}{lcccccc}
\hline \multirow{2}{*}{$\begin{array}{c}\text { Vehicle } \\
\text { Type }\end{array}$} & \multicolumn{2}{c}{ Section-I } & \multicolumn{2}{c}{ Section-II } & \multicolumn{2}{c}{ Section-III } \\
\cline { 2 - 7 } & $\begin{array}{c}\text { Average } \\
\text { Speed } \\
(\mathrm{km} / \mathrm{h})\end{array}$ & $\begin{array}{c}\text { Proportional } \\
\text { Share }\end{array}$ & $\begin{array}{c}\text { Average } \\
\text { Speed } \\
(\mathrm{km} / \mathrm{h})\end{array}$ & $\begin{array}{c}\text { Proportional } \\
\text { Share }\end{array}$ & $\begin{array}{c}\text { Average } \\
\text { Speed } \\
(\mathrm{km} / \mathrm{h})\end{array}$ & $\begin{array}{c}\text { Proportional } \\
\text { Share }\end{array}$ \\
\hline CS & 66.59 & 0.32 & 64.5 & 0.20 & 83.3 & 0.22 \\
CB & 69.80 & 0.07 & 67.0 & 0.06 & 75.1 & 0.10 \\
LCV & 49.80 & 0.03 & 47.6 & 0.07 & 60.1 & 0.04 \\
HCV & 46.70 & 0.04 & 42.1 & 0.07 & 51.9 & 0.08 \\
TW & 50.02 & 0.45 & 45.1 & 0.45 & 56.5 & 0.49 \\
3W & 39.50 & 0.05 & 40.8 & 0.12 & 49.4 & 0.02 \\
B & 50.47 & 0.04 & 45.2 & 0.03 & 66.1 & 0.05 \\
\hline
\end{tabular}

\subsection{Development of Speed Equations and Estimation of Equivalency Units}

The MNLR equation predicts the speed of a vehicle type within a heterogeneous traffic stream. Initially, the speed of vehicle types and their proportional shares are aggregated in 5 minute intervals, establishing a relationship to estimate average speed of the CS. The PCU values of subject vehicle types are identified as regression coefficients of the proposed regression model, as shown in Table 4. The coefficient $a_{1}$ was estimated as the average speed of CS, which is also affected by its own proportional share. The value of coefficient $a_{1}$ was $63 \mathrm{~km} / \mathrm{h}$. The value of $\mathrm{R}^{2}$ for the model is 0.77 . The high $\mathrm{R}^{2}$ value indicates the strength of the model in predicting the speed of CS.

$$
\begin{aligned}
& V_{C S}=a_{1} * n_{C S}+a_{2} *\left[\frac{A_{C S}}{A_{C B}} * n_{C B} * V_{C B}\right]+a_{3} *\left[\frac{A_{C S}}{A_{L C V}} * n_{L C V} * V_{L C V}\right]+a_{4} *\left[\frac{A_{C S}}{A_{H C V}} * n_{H C V} * V_{H C V}\right] \\
& +a_{5} *\left[\frac{A_{C S}}{A_{T W}} n_{T W} * V_{T W}\right]+a_{6} *\left[\frac{A_{C S}}{A_{3 W}} * n_{3 W} * V_{3 W}\right]+a_{7} *\left[\frac{\left.A_{C S} * n_{B} * V_{B}\right]}{A_{B}}\right.
\end{aligned}
$$


where, $a_{2}=\mathrm{PCU}$ of $\mathrm{CB}, a_{3}=\mathrm{PCU}$ of LCV,$a_{4}=\mathrm{PCU}$ of $\mathrm{HCV}, a_{5}=\mathrm{PCU}$ of TW, $a_{6}=\mathrm{PCU}$ of $3 \mathrm{~W}$, $a_{7}=\mathrm{PCU}$ of $\mathrm{B}$.

Table 4 Regression coefficient as PCU value of subject vehicle types

\begin{tabular}{cccc}
\hline Vehicle Type & Coefficients & $\begin{array}{c}\text { PCU } \\
\text { Values }\end{array}$ & $\begin{array}{c}\text { Standard } \\
\text { Error }\end{array}$ \\
\hline BC & $a_{2}$ & 1.56 & 0.15 \\
LCV & $a_{3}$ & 2.69 & 0.42 \\
HCV & $a_{4}$ & 3.83 & 0.65 \\
TW & $a_{5}$ & 0.28 & 0.02 \\
3W & $a_{6}$ & 0.85 & 0.12 \\
B & $a_{7}$ & 6.80 & 1.12 \\
\hline
\end{tabular}

Similarly, Equations 6 and 7 for vehicle types TW and HV were established by using the same set of field data. High $\mathrm{R}^{2}$ values indicate the strength of the models in predicting the speed.

$$
\begin{aligned}
& V_{T W}=50 * n_{T W}+2.82 *\left[\frac{A_{T W}}{A_{C S}} * n_{C S} * V_{C S}\right]+3.82 *\left[\frac{A_{T W}}{A_{C B}} * n_{C B} * V_{C B}\right]+3.43 *\left[\frac{A_{T W}}{A_{L C V}} * n_{L C V} * V_{L C V}\right] \\
& +7.72 *\left[\frac{A_{T W}}{A_{H C V}} * n_{H C V} * V_{H C V}\right]+2.39 *\left[\frac{A_{T W}}{A_{3 W}} * n_{3 W} * V_{3 W}\right]+10.54 *\left[\frac{A_{T W}}{A_{B}} * n_{B} * V_{B}\right] \mathrm{R}^{2}=0.78 \\
& V_{H C V}=46 * n_{H C V}+0.17 *\left[\frac{A_{H C V}}{A_{C S}} n_{C S} * V_{C S}\right]+0.22 *\left[\frac{A_{H C V}}{A_{C B}} n_{C B} * V_{C B}\right]+0.38 *\left[\frac{A_{H C V}}{A_{L C V}} n_{L C V} * V_{L C V}\right] \\
& +0.02 *\left[\frac{A_{H C V}}{A_{T W}} n_{T W} * V_{T W}\right]+0.25 *\left[\frac{A_{H C V}}{A_{3 W}} n_{3 W} * V_{3 W}\right]+1.44 *\left[\frac{A_{H C V}}{A_{B}} * n_{B} * V_{B}\right] \mathrm{R}^{2}=0.76
\end{aligned}
$$

\subsection{Validation of MNLR Speed Models}

Validation of the MNLR speed models was performed using another set of field data obtained from Section-II. The average speed of vehicle types observed on the field section was used validate the values obtained from the models. First, estimated average speeds of CS were compared with the field observed values at varying compositions and volume levels. The two average speeds were plotted against the $45^{\circ}$ line chart, with the comparison shown in Figure 1. The test of significance was performed for comparison and the P-value was obtained and found to be higher than the critical value at $5 \%$ level of significance, which shows no difference between observed and estimated speeds.



Figure 1 Comparison of average speed of CS between estimated and field data 
Similarly, Equation 6 and Equation 7 were also validated using the same set of field data. The two average speeds of TW and HCV were plotted against the $45^{\circ}$ line chart, with the comparisons shown in Figure 2 and Figure 3, respectively. The test of significance was also performed between observed and estimated average speeds of TW and HCV, and also showed no difference between observed and estimated speeds of TW and HCV.

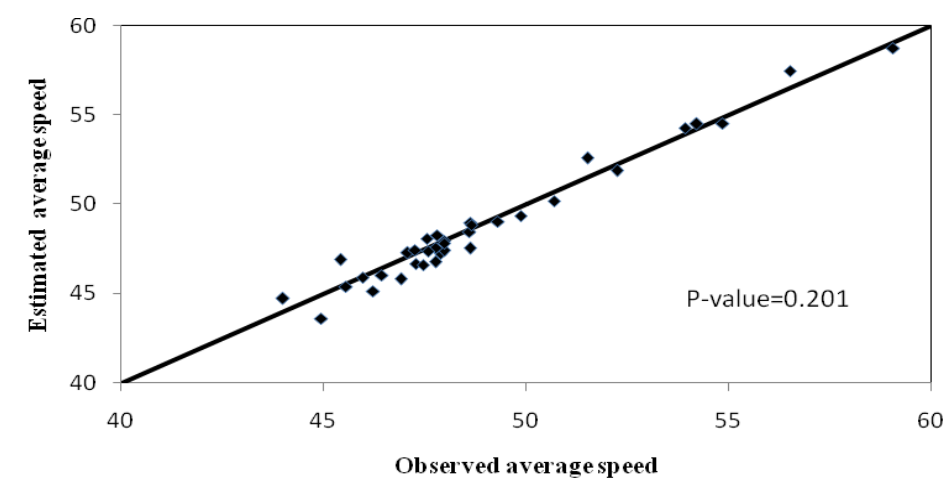

Figure 2 Comparison of average speed of TW between estimated and field data

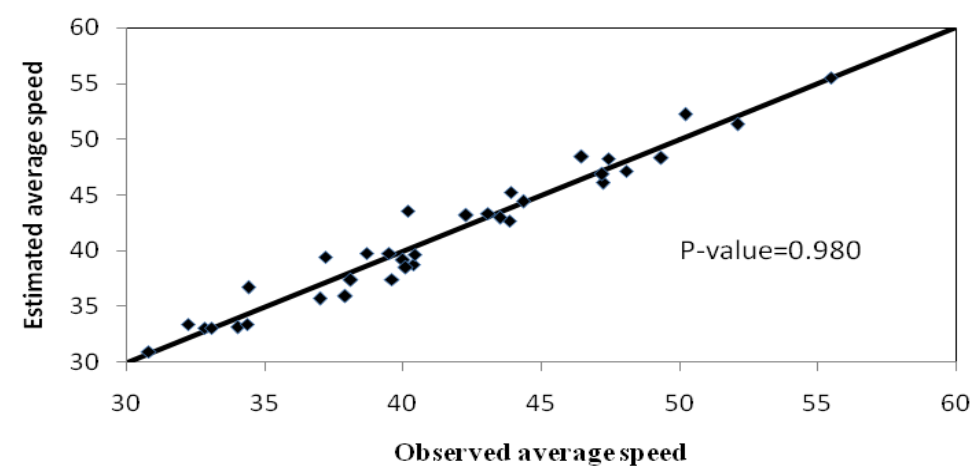

Figure 3 Comparison of average speed of HCV between estimated and field data

\subsection{Estimation of PCU by Dynamic PCU Method}

Chandra et al. (1997) found that the dynamic PCU method is better for estimating the PCU values of different vehicle types under heterogeneous traffic conditions. PCU values for different vehicle types were estimated by taking the speed and area ratios of a CS to subject vehicle measured in Section-I. Equation 8 estimates the PCU value of an $\mathrm{i}^{\text {th }}$ (subject vehicle type) vehicle. This method, proposed by Chandra and Sikdar, is called dynamic PCU and is effectively used for interrupted and uninterrupted traffic conditions.

$$
P C U=\frac{V_{c} / V_{i}}{A_{c} / A_{i}}
$$

where $\mathrm{PCU}_{\mathrm{i}}$ is $\mathrm{PCU}$ of the $\mathrm{i}^{\text {th }}$ vehicle, $V_{c} / V_{i}$ is speed ratio of the car to the $\mathrm{i}^{\text {th }}$ vehicle and $A_{c} / A_{i}$ is space ratio of the car to the $\mathrm{i}^{\text {th }}$ vehicle.

The PCU values estimated by the MNLR method were compared with the values obtained by the dynamic PCU method and are shown in Figure 4. The traffic volume data collected at Section-I was converted into PCU/hr using PCU values obtained by the dynamic PCU method. Figure 5 shows the observations and flow where flow was converted into PCU/hr using the 
MNLR and dynamic PCU methods. The plot shows that the maximum traffic flow is 1728 PCU/hr where flow was converted into PCU/hr using the MNLR method, while the maximum traffic flow is $1628 \mathrm{PCU} / \mathrm{hr}$ where flow was converted into PCU/hr using the dynamic PCU method. The maximum traffic flow is $1710 \mathrm{PCU} / \mathrm{hr}$ where flow was converted into PCU/hr using PCUs suggested by IRC 64-1990. The maximum flow obtained by the MNLR method is almost on par with the maximum flow when PCU suggested by IRC is used. Hence it is justified that the PCU by MNLR method is more realistic and logical compared to the dynamic method.

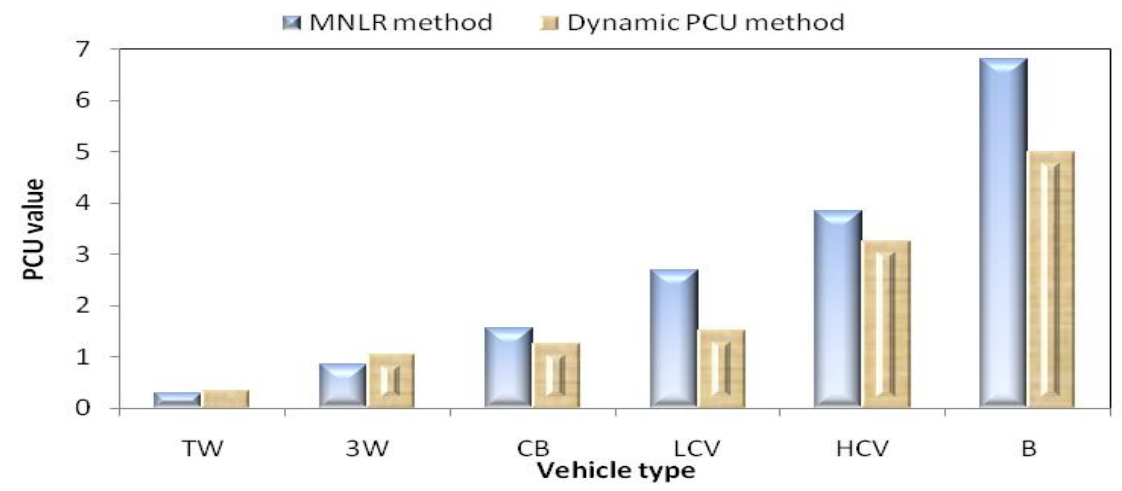

Figure 4 PCU estimated using MNLR method and dynamic method for subject vehicle types

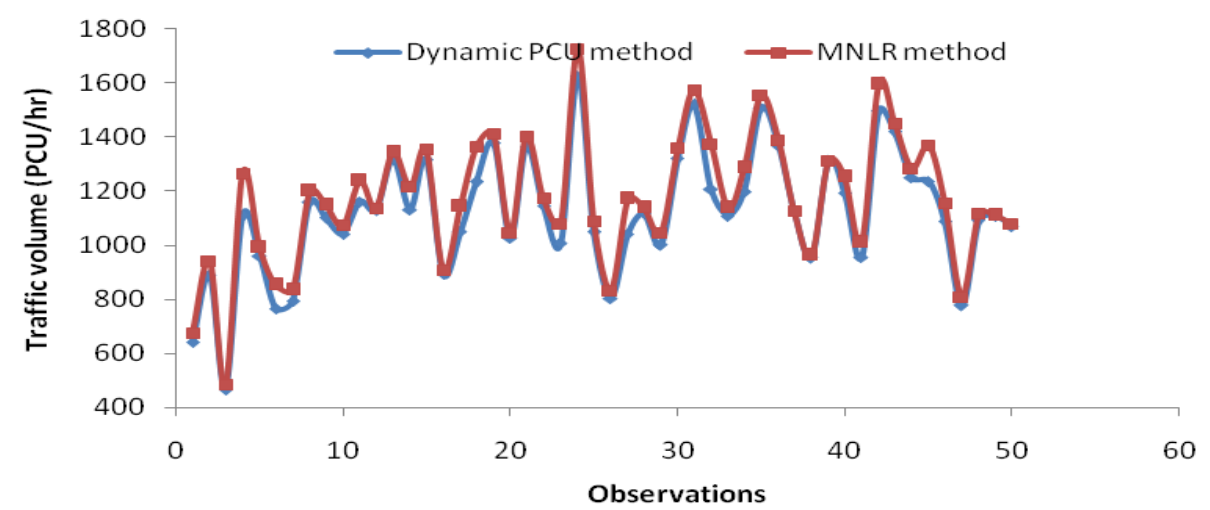

Figure 5 Comparison of traffic volume using both methods

\subsection{Six-Lane Divided Highway}

The multiple non-linear regression approach can be used for estimating the equivalency units on six-lane and eight-lane divided highways. The PCU values for different vehicle types at Section-III are estimated using the MNLR model. The equivalency units of different vehicle types at Section-III are shown in Table 5. The obtained equivalency units are realistic and logical values.

Table 5 Equivalency units of different vehicles at Section-III

\begin{tabular}{llrc}
\hline Vehicle Type & PCU & TwU & HCVU \\
\hline CS & 1.000 & 2.624 & 0.205 \\
BC & 1.660 & 3.246 & 0.504 \\
TW & 0.342 & 1.000 & 0.086 \\
LCV & 2.239 & 3.245 & 0.908 \\
3W & 1.221 & 2.126 & 0.227 \\
HCV & 3.828 & 7.413 & 1.000 \\
BUS & 6.181 & 10.246 & 2.088 \\
MAV & 7.862 & 15.346 & 2.362 \\
\hline
\end{tabular}




\section{CONCLUSION}

The unique multiple non-linear approach can be used to accurately estimate the equivalency units of individual vehicle types under heterogeneous traffic conditions. The PCU values estimated using the MNLR method are found to be realistic and logical under heterogeneous traffic flow conditions. The dynamic method considers the speed ratio and an area ratio of vehicle types but ignores the effect of vehicle composition under heterogeneous traffic conditions. However, the MNLR method considers the effect of vehicle composition for estimating the PCU values. From the results, it is concluded that the PCU by MNLR method is more realistic and logical compared to the dynamic method. The accuracy of the proposed model was checked by collecting field data at one additional section of four-lane road. The speeds obtained from the models were found in good agreement with observed speeds in the field, i.e., both the speeds are along a $45^{\circ}$ line. The test of significance also indicated there was no difference between estimated and observed speeds at $5 \%$ level of significance. These models are useful in estimating the equivalency unit of a vehicle at a given volume and composition of a traffic stream. The multiple non-linear approach was also used for estimating the equivalency units on a six-lane divided highway. The results showed that the multiple non-linear approach can be used for estimation of equivalency units for vehicle types under mixed traffic conditions. But the present study has practical difficulties related to field data because the traffic composition of all vehicle types cannot be obtained under controlled conditions. The study will be continued to observe the variation in PCU with respect to composition of each vehicle type using a simulation technique.

\section{REFERENCES}

Arasan, V.T., Arkatkar, S.S., 2010. Micro-simulation Study of Effect of Volume and Road Width on PCU of Vehicles under Heterogeneous Traffic. Journal of Transportation Engineering, Volume 136(12), pp. 1110-1119

Cao, N.Y., Sano, K., 2012. Estimating Capacity and Motorcycle Equivalent Units on Urban Roads in Hanoi, Vietnam. Journal of Transportation Engineering, Volume 138(6), pp. 776-785

Chandra, S., Kumar, U., 2003. Effect of Lane Width on Capacity under Mixed Traffic Conditions in India. Journal of Transportation Engineering, Volume 129(2), pp. 155-160

Chandra, S., Zala, L.B., Kumar, V., 1997. Comparing the Methods of Passenger Car Unit Estimation. Journal of Institution of Engineer, Volume 78, pp. 13-16

Chandra, S., Kumar, V., Sikdar, P.K., 1995. Dynamic PCU and Estimation of Capacity of Urban Roads. Indian Highways, Volume 23(4), pp. 17-28

Cunagin, W.D., Messer, C.J., 1982. Passenger Car Equivalents for Rural Highways. Transportation Research Record, No. 905, pp. 61-68

Huber, M.J., 1982. Estimation of Passenger-car Equivalents of Trucks in Traffic Stream. Transportation Research Record, No. 869, pp. 60-70

IRC 64, 1990. Guidelines for Capacity of Roads in Rural Areas. Indian Road Congress, New Delhi, India

Karim, M.R., Anand S., Sekhar, S.V.C., 1999. Development of Passenger Car Unit (PCU) Values for Malaysia. Journal of the Eastern Asia Society for Transportation Studies, Volume 3(3), pp. 73-80

St. John, A.D., Kobett, D.R., 1978. Grade Effects on Traffic Flow Stability and Capacity. Technical Report NCHRP Report 185, Washington, DC

Transportation Research Board, 1965. Special Report 87. Highway Capacity Manual, Washington, DC 
Webster, N., Elefteriadou, L., 1999. A Simulation Study of Truck Passenger Car Equivalents (PCE) on Basic Freeway Sections. Journal of Transportation Research, Part B, Volume 33 (5), pp. 323-336 